\title{
ONE-DIMENSIONAL STOCHASTIC MODELS WITH OPEN BOUNDARIES: INTEGRABILITY, APPLICATIONS AND $q$-DEFORMED KNIZHNIK-ZAMOLODCHIKOV EQUATIONS
}

\author{
CALEY FINN
}

(Received 30 June 2015; first published online 19 August 2015)

2010 Mathematics subject classification: primary 82B23; secondary 82C22, 60K25, $20 \mathrm{C} 08$.

Keywords and phrases: statistical mechanics, exactly solvable models, queueing systems, Hecke algebras.

This thesis contains work on three separate topics, but with common themes running throughout. These themes are drawn together in the asymmetric exclusion process (ASEP) - a stochastic process describing particles hopping on a one-dimensional lattice [5]. The open-boundary ASEP is set on a finite-length lattice, with particles entering and exiting at both boundaries. The transition matrix of the open-boundary ASEP provides a representation of the two-boundary Temperley-Lieb algebra, and the integrability of the system allows the diagonalisation of the transition matrix through the Bethe ansatz method.

We study the ASEP in the reverse-bias regime, where the boundary injection and extraction rates oppose the preferred direction of flow in the bulk. We find the exact asymptotic relaxation rate along the coexistence line by analysing solutions of the Bethe equations. The Bethe equations are first solved numerically; then the form of the resulting root distribution is used as the basis for an asymptotic analysis. The reverse bias induces the appearance of isolated roots, which introduces a modified length scale in the system. We describe the careful treatment of the isolated roots that is required in both the numerical procedure and in the asymptotic analysis (see [2]).

The second topic of this thesis is the study of a priority queueing system, modelled as an exclusion process with moving boundaries. We call this model the prioritising exclusion process (PEP). In the PEP, the hopping of particles corresponds to highpriority customers overtaking low-priority customers in order to gain service sooner. The PEP is related to a priority queueing model first introduced by Kleinrock [4], and more recently studied in the context of healthcare queues [6]. Although the PEP is not integrable, techniques from the ASEP allow calculation of exact density profiles

Thesis submitted to the University of Melbourne in December 2014; degree approved on 2 March 2015; principal supervisor Jan de Gier and co-supervisors Richard Brak and Mark Sorrell.

(c) 2015 Australian Mathematical Publishing Association Inc. 0004-9727/2015 \$16.00 
in certain phases, and the calculation of approximate average waiting times when the expected queue length is finite (see [1]).

The final topic of this thesis is a study of polynomial solutions of a $q$-deformed Knizhnik-Zamolodchikov ( $q \mathrm{KZ}$ ) equation with mixed boundaries. The $q \mathrm{KZ}$ equation studied here is given in terms of the one-boundary Temperley-Lieb algebra, and its solutions have a factorised form in terms of Baxterised elements of the type $B$ Hecke algebra [3]. We find an integral form for certain components of the $q \mathrm{KZ}$ solution, along with a factorised expression for a generalised sum rule. The representation of the Temperley-Lieb algebra that we study is related to the $O(n)$ Temperley-Lieb loop model, and a specialisation of the sum rule gives the normalisation of the ground state vector for the $O(n=1)$ model.

\section{References}

[1] J. de Gier and C. Finn, 'Exclusion in a priority queue', J. Stat. Mech. 2014 (2014), P07014.

[2] J. de Gier, C. Finn and M. Sorrell, 'The relaxation rate of the reverse-biased asymmetric exclusion process', J. Phys. A $\mathbf{4 4}$ (2011), 405002.

[3] J. de Gier and P. Pyatov, 'Factorised solutions of Temperley-Lieb qKZ equations on a segment', Adv. Theor. Math. Phys. 14 (2010), 795-877.

[4] L. Kleinrock, 'A delay dependent queue discipline', Naval Res. Logist. Quart. 11(3-4) (1964), 329-341.

[5] F. Spitzer, 'Interaction of Markov processes', Adv. Math. 5(2) (1970), 246-290.

[6] D. A. Stanford, P. Taylor and I. Ziedins, 'Waiting time distributions in the accumulating priority queue', Queueing Syst. 77(3) (2014), 297-330.

\section{CALEY FINN, Department of Mathematics and Statistics,}

University of Melbourne, Victoria 3010, Australia

e-mail: caleyreuben@gmail.com 